\title{
Anti-diabetic drug discovery using bioactive compounds: Molecular docking insights
}

John Oluwafemi Teibo 1, 2, , Samuel Abidemi Bello ${ }^{1}$, Oluwaseun Abraham Adebisi ${ }^{1}$, Jeremiah Olorunjuwon Olugbami ${ }^{1}$ and Titilade Kehinde Ayandeyi ${ }^{3}$

${ }^{1}$ Department of Biochemistry, University of Ibadan, Ibadan, Oyo State, Nigeria.

${ }^{2}$ Department of Biochemistry and Immunology, Ribeirao Preto Medical School, University of Sao Paulo, Ribeirao Preto, SPBrazil.

${ }^{3}$ Department of Maternal-Infant and Public Health Nursing, University of São Paulo at Ribeirão Preto, College of Nursing, Ribeirão Preto, SP-Brazil.

GSC Biological and Pharmaceutical Sciences, 2021, 14(03), 175-178

Publication history: Received on 11 January 2021; revised on 10 March 2021; accepted on 12 March 2021

Article DOI: https://doi.org/10.30574/gscbps.2021.14.3.0048

\begin{abstract}
Diabetes mellitus is a metabolic disorder that has become a global health problem. About 500 million people were estimated to be living with diabetes in 2018 with about 20 million in Africa and 2 million cases in Nigeria. Bioactive compounds offer an advanced starting point in the search for highly specific and potent modulator of bimolecular function as well as novel drugs, which can be studied with more precision by using computer aided drug design (CADD). Molecular docking employed for predicting the interactions between receptor and ligands is an integral aspect in drug discovery. The main objective is to attain ligand-receptor complex with optimized conformation and with the intention of possessing less binding free energy.
\end{abstract}

Several studies have used this method to explore the potency of bioactive compounds to predict better alternatives in the search for an anti-diabetic drug with very effective therapeutic role and minimal side effects. This has been carried out by using several compounds such as Quercetin, against endogenous targets such as Glycogen phosphorylase, Peroxisome Proliferator-activated Receptor (PPAR)-y, Glucokinase, Protein Tyrosine Phosphatase 1-beta (PTP-1B), GLUT4, etc. In Silico tools such as Protein Database (PDB), GenBank and softwares such as Autodock and modeller are of major importance to these studies.

The paper seeks to examine bioactive compounds basically quercetin that have been successfully identified through molecular docking and their molecular targets as well as recent advances in the use of molecular docking in the novel discovery and explanation of mechanisms of actions of some bioactive compounds in anti-diabetic drug discovery.

Keywords: Diabetes mellitus; Molecular Docking; Bioactive compounds; Quercetin; Computer-Aided Drug Discovery and Design (CADD).

\section{Introduction}

Diabetes Mellitus (DM) is a metabolic disorder characterized by the presence of chronic hyperglycemia. It was reported that 366 million people had DM in 2011, and by 2030 this number is estimated to almost around 552 million [1]. The menace of DM and the rapid increase in number of cases worldwide has led to a pool of research on its causes and treatments. No cure has yet been found for the disease; however, treatment modalities include improved lifestyle changes, orally administered hypoglycemic agents, and use of metformin (an insulin sensitizer) [2]. Several studies have been carried out in vivo using bioactive compounds in natural plants (such as quercetin and yohimbine) in a search for

\footnotetext{
* Corresponding author: John Oluwafemi Teibo, teiboluwafemi@gmail.com

Department of Biochemistry and Immunology, Ribeirao Preto Medical School, University of Sao Paulo, Ribeirao Preto, SP-Brazil.
} 
the cure to DM. Biochemical studies have revealed that these bioactive compounds through different mechanisms have various activities in the human body such as antioxidant and antidiabetic functions $[3,4]$.

Computer-aided drug designs (CADD) are playing an increasing role in drug discovery and are critical in the costeffective identification of promising drug candidates [5]. Molecular docking employed for predicting the interactions between receptor and ligands is an integral aspect in drug discovery. The main objective is to attain ligand-receptor complex with optimized conformation and with the intention of possessing less binding free energy.

\section{Diabetes Mellitus (DM)}

The etiology of DM can vary greatly but always include defects in either insulin secretion, response or both at some point in the course of the disease [6]. DM can be classified into four classes which are; type 1 diabetes, type 2 diabetes, gestational diabetes, and other specific types [7].

\subsection{Causes of Diabetes Mellitus}

DM is a chronic metabolic disorder which is either immune-mediated as seen in Type 1 diabetes, insulin resistance as in Type 2 Diabetes, gestational as observed in pregnancy or due to other factors such as infections, environment, genetic defects and certain drugs [6].

Insulin and insulin receptors play a key role in the etiology of all forms of DM. Insulin exhibits a multitude of effects in many tissues, with liver, muscle, and adipose tissue being the most important target organs for insulin action. The basic physiological function of insulin is promoting the synthesis of carbohydrates, proteins, lipids, and nucleic acids. Insulin functions in stimulation of transport of glucose across muscle and adipocyte cell membranes, hepatic glycogen synthesis regulation, and glycogenolysis and gluconeogenesis inhibition [8]. Hence impairment of insulin function or response leads to a myriad of metabolic aberrations.

\subsection{Treatment}

Oral hypoglycemics have been of help in the maintenance of blood glucose level in diabetics through distinct mechanisms [9]. Sulfonylureas and non-sulfonylureas ensure normal glycemia by stimulating endogenous insulin secretion; alpha-glucosidase inhibitors work by delaying absorption of saccharides in the gut while thiazolidinediones (TZDs) maintain normal glycemia by stimulating insulin sensitivity majorly by enhancing peripheral glucose removal, and suppressing glucose production by the liver. Metformin is another major drug used in the management of diabetes; it works by downregulating hepatic gluconeogenesis while at times also increasing peripheral glucose mobilization and disposal [10]. Type I diabetes mellitus patients also rely on synthetic insulin injection.

\section{Molecular Docking Studies}

Molecular docking uses specific algorithm to find the best ligand molecule that fits within the active site of a target molecule possessing best possible pose. This search algorithm utilizes an energy scoring function suggesting the ligand molecule with lowest binding energy has the highest affinity for receptor binding [11]. In the experiment reported by [12] all the selected ligand molecules- Aegeline, Gallic acid, Mangiferin and Quercetin interacted with the target molecule with $-5.622 \mathrm{Kcal} / \mathrm{mol},-6.213 \mathrm{Kcal} / \mathrm{mol},-6.191 \mathrm{Kcal} / \mathrm{mol}$ and $-6.496 \mathrm{Kcal} / \mathrm{mol}$ binding energy respectively. Quercetin with the lowest binding energy has the highest affinity for GSK3B binding and the highest binding energy of Aegeline suggests lowest affinity of interaction with GSK3B. Aegeline formed hydrogen bonds with 3 amino acid residues (VAL135, PR0136 and ARG141) of the target molecule, Gallic acid formed 4 hydrogen bonds (2×ILE62, VAL135, ARG141), Mangiferin formed 6 hydrogen bonds (2×ASP200, ASP133, ASP181, LYS83, LYS183) and Quercetin formed again 4 hydrogen bonds (2×ASP200, ARG141, VAL135) as Gallic acid within the active site of GSK3B. Aegeline and Gallic acid each interacted with 6 residues within the binding pocket of the target molecules whereas Mangiferin and Quercetin interacted with 10 and 8 residues respectively.

\subsection{Drug-likeness Property}

Lipinski's rule of five provides indication about the solubility, bioavailability and permeability of the drug molecule of interest. This rule assists in the shift of phase I trial to phase II trial of an investigational new drug with the help of computational approach that reduces both time and cost of the experiment [13]. TPSA or topological polar surface area is again associated with absorption and permeability of the drug molecule. Any candidate with higher TSPA value often results in poor permeability in biological system. Furthermore, higher drug score and drug likeness score indicate the best possibility of a candidate molecule to have the all the physicochemical parameters within range. 
Mangiferin showed the highest TSPA value (201.28) and Aegeline showed the lowest TSPA value (58.56). Gallic acid and Quercetin showed moderate level of TSPA value- 201.28 and 127.4 respectively. Quercetin exhibited better drug likeness score (1.6) and drug score (0.3) after Aegeline which exhibited likeness score of 2.0 and drug score of 0.83.

\subsection{ADME/T Test}

All the molecules are impermeable to blood brain barrier offering no chance to cause damage to brain. Every molecule showed high absorption ability in intestine and didn't show any indication of carcinogenicity. Only Gallic acid showed colorectal tissue permeability. Cytochrome P450 (CYP) family of enzymes play major roles in drug metabolism, excretion and drug-drug interaction. Inhibition of any of these enzymes may cause unusual effects like slow degradation rate of the drug molecule, accumulation in the body and slow excretion [14]. Aegeline and quercetin are potent inhibitors of CYP450 3A4 and again quercetin is another inhibitor of CYP450 1A2. Gallic acid and mangiferin are not any inhibitors of any type of cytochrome P450 enzyme. Only gallic acid showed high degree of biodegradability among all the ligand molecules. Mangiferin showed the possibility to induce mutation as it positively indicated AMES toxicity.

\section{Conclusion}

Thousands of plants in nature have been reported to have antidiabetic activity. They have different mode of actions and deploy their effects by variety of mechanisms [15]. Quercetin could be a great candidate of antidiabetic agent against type II diabetes since commercially available treatments are not cost effective and employs some other complications. Moreover, other molecules also performed well indicating their potentiality to be used as antidiabetic agent. A well designed and targeted research can help us finding a potent and natural source of antidiabetic agent. Hopefully, molecular docking studies will enable researchers discover potent antidiabetic agents involved in the novel pathways also explanation of mechanisms of actions of these bioactive compounds with an overall aim in propelling anti-diabetic drug discovery will be effective.

\section{Compliance with ethical standards}

\section{Acknowledgments}

We appreciate all researchers on the pathway to novel anti-diabetic drug discovery.

\section{Disclosure of conflict of interest}

There is no conflict of interest among the authors

\section{References}

[1] Chen L, Magliano DJ, Zimmet PZ. The worldwide epidemiology of type 2 diabetes mellitus: present and future perspectives. Nature reviews endocrinology. Nature Reviews Endocrinology 2011; 8(4):228-36

[2] Olokoba AB, Obateru OA, Olokoba LB. Type 2 Diabetes Mellitus: A Review of Current Trends. Oman Med J. 2012; 27(4): 269-273.

[3] Teibo J, Bello S, Olagunju A, Olorunfemi F, Ajao O, Fabunmi O. Functional foods and bioactive compounds: Roles in the prevention, treatment and management of neurodegenerative diseases. GSC Biological and Pharmaceutical Sciences. 2020; 11(2): 297-313.

[4] Olagunju AS, Olorunfemi FG, Teibo OJ, Bello SA, Fabunmi TE, Ajao OM. Functional-Foods Use: A Novel Dietary Regimen for Type 2 Diabetes Treatment and Management IJISRT 2020;5(6):340-345

[5] Brogi, S. Ramalho, T. C, Medina-Franco, J. L, Kuca, K, Valko, M., eds. In Silico Methods for Drug Design and Discovery. Lausanne: Frontiers Media SA. (2020) ISBN: 978-2-88966-057-5

[6] Baynes HW. Classification, Pathophysiology, Diagnosis and Management of Diabetes Mellitus. J Diabetes Metab. 2015; 6: 541.

[7] Sicree R, Shaw J and Zimmet P. The Global Burden. Diabetes and Impaired Glucose Tolerance. Prevalence and Projections. In: Gan, D. ed. Diabetes Atlas, 3rd edn. Brussels: International Diabetes Federation. 2006; 16-103.

[8] Piero NM, Joan MN, Cromwell KM, Joseph NJ, Wilson NM, et al. (2011) Hypoglycemic Activity of Some Kenyan Plants Traditionally used to Manage Diabetes Mellitus in Eastern Province. J Diabetes Metab 2:155. 
[9] Inzucchi SE. Oral antihyperglycemic therapy for type 2 diabetes: scientific review. JAMA. 2002; 287(3): 360-372.

[10] Curtis LT. New technologies and therapies in the management of diabetes. American journal of managed care, 13(2 suppl): S47-S54. 2007.

[11] Guedes IA, de Magalhães CS, Dardenne LE. Receptor-ligand molecular docking; Biophysical reviews. 2014; 6(1): 75-87.

[12] Prottoy NL, Ullah MA, Sarkar B, Hossain S, Boby AS, Araf Y. Molecular docking and pharmacological property analysis of antidiabetic agents from medicinal plants of Bangladesh against Type 11 Diabetes: A computational approach; PharmaTutor; 2019; 7(9); 6-15

[13] Pollastri MP. Overview on the Rule of Five. Current protocols in pharmacology. 2010; 49(1): 9-12.

[14] Newbert DW, Russell DW. Clinical importance of the cytochromes P450; The Lancet. 2002; 360(9340): 11551162.

[15] Marles RJ, Farnsworth NR. Antidiabetic plants and their active constituents; Phytomedicine. 1995; 2(2): 137-189. 